\title{
Reflets
}

Revue d'intervention sociale et communautaire

\section{Être homosexuel et d'origine libanaise en contexte montréalais : identifications ethno-sexuelles multiples, complexes et variables}

\section{To be Queer and Lebanese in Montreal: Multiple, Complex and Variable Ethno-Sexual Identities}

\section{Marianne Chbat}

Volume 23, numéro 1, printemps 2017

Militantisme et changement social. Partie 1

URI : https://id.erudit.org/iderudit/1040752ar

DOI : https://doi.org/10.7202/1040752ar

Aller au sommaire du numéro

Éditeur(s)

Reflets, Revue d'intervention sociale et communautaire

ISSN

1203-4576 (imprimé)

1712-8498 (numérique)

Découvrir la revue

Citer cet article

Chbat, M. (2017). Être homosexuel et d'origine libanaise en contexte montréalais : identifications ethno-sexuelles multiples, complexes et variables. Reflets, 23(1), 148-173. https://doi.org/10.7202/1040752ar
Résumé de l'article

En nous inspirant des résultats de notre mémoire, nous rendons compte dans cet article des diverses façons dont certaines personnes LGBTQ+ d'origine libanaise, vivant à Montréal et impliquées au sein d'un organisme militant pour les droits des minorités sexuelles et racisées, articulent et négocient leurs identifications ethno-sexuelles. À partir d'un cadrage intersectionnel qui prend en considération l'imbrication de divers axes de structuration sociale que sont le genre, la classe, la sexualité, l'ethnicité, l'âge, etc., les résultats de cette recherche suggèrent qu'il existe une multiplicité de discours identitaires, ces derniers déstabilisant, dans certains cas, ou (re)produisant, dans d'autres, les représentations normatives de l'identité homosexuelle hégémonique fortement véhiculée actuellement en contexte occidental.
Tous droits réservés (C) Reflets, Revue d'intervention sociale et communautaire, 2017
Ce document est protégé par la loi sur le droit d’auteur. L'utilisation des services d'Érudit (y compris la reproduction) est assujettie à sa politique d'utilisation que vous pouvez consulter en ligne. 


\title{
Être homosexuel et d'origine libanaise en contexte montréalais : identifications ethno-sexuelles multiples, complexes et variables
}

\author{
Marianne Chbat \\ Doctorante en sciences humaines appliquées \\ Université de Montréal \\ marianne.chbat@umontreal.ca
}

\section{Résumé}

En nous inspirant des résultats de notre mémoire, nous rendons compte dans cet article des diverses façons dont certaines personnes LGBTQ+ d'origine libanaise, vivant à Montréal et impliquées au sein d'un organisme militant pour les droits des minorités sexuelles et racisées, articulent et négocient leurs identifications ethno-sexuelles. À partir d'un cadrage intersectionnel qui prend en considération l'imbrication de divers axes de structuration sociale que sont le genre, la classe, la sexualité, l'ethnicité, l'âge, etc., les résultats de cette recherche suggèrent qu'il existe une multiplicité de discours identitaires, ces derniers déstabilisant, dans certains cas, ou (re)produisant, dans d'autres, les représentations normatives de l'identité homosexuelle hégémonique fortement véhiculée actuellement en contexte occidental.

Mots clés : homosexualité, queer, ethnicité, Liban, intersectionnalité, identité, gais et lesbiennes, diaspora, Montréal

\section{To be Queer and Lebanese in Montreal: Multiple, Complex and Variable Ethno-Sexual Identities}

\section{Abstract}

Drawing on the results of our thesis, this article reports on the ways in which some of LGBTQ+ people from Lebanese descent, living in Montreal and whom are involved in a militant organization for the rights of sexual and racialized minorities, articulate and 
negotiate their ethno-sexual identifications. Inspired by an intersectional framing that takes into account the overlapping effects of various axes of social structures such as gender, class, sexuality, ethnicity, age, etc., the results of this research suggest, among other things, that there are different ways of representing oneself and being homosexual, and that individuals sharing non-normative sexualities do not necessarily inscribe themselves in the hegemonic gay discourse currently dominant within Western context.

Key words: homosexuality, queer, ethnicity, Lebanon, intersectionality, identity, gays and lesbians, diaspora, Montreal

Aujourd'hui, c'est devenu un lieu commun d'affirmer que la mondialisation accrô̂t la circulation et la formation des identités homosexuelles, plus particulièrement celle des modèles occidentaux ${ }^{1}$ qui subjuguent d'autres conceptions et d'autres expériences (Calixte, 2005; Chahine, 2008; Chbat, 2011; Khalaf et Gagnon, 2006; Massad, 2002). En effet, si cette prolifération des identités homosexuelles et des savoirs sur celles-ci, tant au niveau local que mondial, est une évidence, encore faut-il appréhender ces identifications et ces savoirs en les situant dans leurs contextes culturels, géographiques, économiques, politiques, historiques et sociaux d'émergence et cerner les tensions et les possibilités qu'engendre leur transnationalisation (Luibhéid, 2005).

"...cet article souhaite
mettre en lumière les
différents facteurs qui
modulent et appuient la
construction identitaire
de certaines personnes
queer racisées, particu-
lièrement leur implica-
tion, à divers degrés,
au sein d'organismes
militant pour les droits
des minorités sexuelles et
raciales,... "

Inspiré par les résultats de notre mémoire, cet article souhaite mettre en lumière les différents facteurs qui modulent et appuient la construction identitaire de certaines personnes queer racisées, particulièrement leur implication, à divers degrés, au sein d'organismes militant pour les droits des minorités sexuelles et raciales, tels que Helem. Comme il s'agit d'une population qui a reçu de plein fouet l'islamophobie généralisée de l'après 11 septembre 2001, il devient particulièrement important de comprendre les stratégies que ces individus déploient pour articuler leurs discours identitaires. En d'autres termes, nous tenterons de rendre compte des diverses manières à travers lesquelles ces individus articulent leurs identifications ethno-sexuelles dans un contexte où il y a une augmentation de la stigmatisation envers les personnes racisées - comme en témoigne la prépondérance des figures du musulman sexiste et homophobe, de la musulmane opprimée, du gai et de la lesbienne dans le placard par peur de représailles, etc. (Chbat, 2011). 
Après un survol critique des recherches s'étant penchées sur les questions d'homosexualité au Moyen-Orient et en contexte diasporique et migratoire, nous présenterons le cadre d'analyse de la recherche, sa méthodologie ainsi que l'analyse de ses résultats.

\section{Problématique}

\section{Immigration des personnes queer racisées : imposition d'un modèle (homo)sexuel et racial dominant}

Avant les années 1990, la revendication d'une identité " gaie " était souvent associée à l'homme blanc, occidental, de classe moyenne (Luibhéid, 2005; Randazzo, 2005). Cependant, la mondialisation et la migration de diverses personnes queer ${ }^{2}$ racisées $^{3}$

"..." l'identité gaie» a longtemps été perçue comme une conséquence de la modernisation et de l'occidentalisation. Or, les immigrantes et immigrants qui revendiquent cette identité ou toute forme de sexualité non normative ne peuvent être réduits à de simples modèles d'assimilation de la culture occidentale dominante, leurs revendications identitaires émergeant plutôt des dynamiques de pouvoir complexes qui sinscrivent à lintersection de la migration et des transformations culturelles que cette dernière provoque souvent...» ont fortement contribué à la transformation des représentations et modèles occidentaux en termes de sexualités non normatives (Appadurai, 1996). En effet, « l'identité gaie » a longtemps été perçue comme une conséquence de la modernisation et de l'occidentalisation. Or, les immigrantes et immigrants qui revendiquent cette identité ou toute forme de sexualité non normative ne peuvent être réduits à de simples modèles d'assimilation de la culture occidentale dominante, leurs revendications identitaires émergeant plutôt des dynamiques de pouvoir complexes qui s'inscrivent à l'intersection de la migration et des transformations culturelles que cette dernière provoque souvent (Chahine, 2008; Luibhéid, 2005; Roy, 2013).

Bien que l'adoption d'une politique du multiculturalisme en 1971 et l'adoption de la Charte canadienne des droits et libertés aient façonné les perceptions locales et mondiales du Canada en tant qu'endroit accueillant et respectueux des choix individuels et de la diversité sexuelle et culturelle, l'immigration au Canada pour les personnes homosexuelles, et particulièrement pour celles qui demandent l'asile, demeure une expérience ambivalente 
et complexe (Gosine, 2008; Jimenez, 2004; Roy, 2013). En effet, ces dernières sont souvent dans l'obligation de dépeindre leur pays d'origine comme un lieu d'oppression et de barbarie afin de faire valoir leur demande (Cantú, 2005). L'Occident devient alors le seul endroit privilégié vers lequel les immigrantes et immigrants homosexuels peuvent se tourner. Ce raisonnement binaire, qui représente, d'une part, l'Occident comme le seul endroit démocratique de liberté et, d'autre part, les « autres » (les pays non occidentaux) comme des lieux monolithiques d'oppression, occulte toutes les formes de subjectivités et d'identifications gaies et lesbiennes dans les pays non occidentaux. De plus, il fait montre d'une violence symbolique à l'égard des migrantes et migrants qui doivent souvent rejeter catégoriquement tout ce qui est associé à leur pays d'origine et s'approprier des postures identitaires et une façon de nommer et de vivre leur sexualité non normative selon des modèles qui ne sont pas toujours adaptés à leurs contextes familiaux et sociaux (Cantú, 2005; Chbat, 2011).

En mettant la lumière sur ce désaveu imposé à la personne homosexuelle face à son pays d'origine, il devient possible de réfléchir sur les dynamiques impérialistes et mondialisées qui sont cachées, mais qui sont intrinsèquement liées aux politiques de contrôle de l'immigration en Occident. En effet, cette opposition hiérarchique entre l'Occident et les " autres " déresponsabilise la société d'établissement de ses échecs politiques (notamment par rapport à "l'intégration » des immigrantes et immigrants) et permet de dissimuler des barrières structurelles persistantes de racisme systémique (Bilge, 2010).

"...bien que les
personnes queer
racisées soient main-
tenant admissibles à
l’immigration, elles
restent souvent confron-
tées à d'autres bar-
rières légales et sociales
qui contraignent leur
migration et affectent
nécessairement leurs
identifications ethno-
sexuelles."

Ainsi, bien que les personnes queer racisées soient maintenant admissibles à l'immigration, elles restent souvent confrontées à d'autres barrières légales et sociales qui contraignent leur migration et affectent nécessairement leurs identifications ethno-sexuelles. En effet, les personnes qui quittent leur pays d'origine ne retrouvent pas nécessairement la liberté recherchée ou espérée en terre d'établissement (Acosta, 2008; Luibhéid, 2005; Roy, 2013). Plusieurs d'entre elles se retrouvent dans des communautés homophobes, et certaines avancent qu'elles ne peuvent pas compter sur le soutien des membres de leur communauté ethnique en contexte diasporique (Acosta, 2008; Randazzo, 2005). De plus, leur âge, leur genre, leur classe et leur ethnicité sont des facteurs importants qui influencent leurs relations avec 
les membres de leur communauté ethnique ainsi que leurs relations avec les membres des communautés queer majoritairement blanches en terre d'établissement (Randazzo, 2005; Roy, 2013). Ces personnes vivent donc souvent des marginalisations multiples. Cependant, les diverses oppressions potentiellement vécues ne s'incarnent pas seulement dans une dynamique de pouvoir négatif qui interdit ou rejette le sujet, le niant de toute agentivité, mais peuvent également donner lieu à des subjectivités porteuses d'un certain pouvoir positif et productif (Collins, 2000; Foucault, 1976; Roy, 2013).

\section{Différentes façons de négocier sa sexualité non normative (sortir du modèle dominant de l'homosexualité occidentale)}

Plusieurs études portant sur les parcours migratoires de certaines personnes queer racisées éclairent bien les différentes formes de marginalisation qu' elles peuvent rencontrer (Acosta, 2008; Cantú, 2005; Chahine, 2008; Chbat, 2011; Manalansan IV, 2005; Roy, 2013; Siraj, 2006). Dans plusieurs cas, la dépendance financière des personnes queer envers leurs parents les oblige à tenir des discours distincts et parfois antagonistes par rapport à leur sexualité. En effet, plusieurs personnes qui vivent encore avec leurs parents ou qui sont dépendantes financièrement d'eux se sentent souvent contraintes d'effacer leur identification (homo)sexuelle afin de maintenir des relations cordiales avec leurs familles, et ce, jusqu'à l'obtention d'une certaine indépendance financière (Acosta, 2008; Chbat, 2011). Cependant, la dépendance économique peut également s'inverser en contexte migratoire, la famille devenant dépendante de l'aide financière de la personne queer. Cette situation tendra à accroître la tolérance à l'endroit de la différence sexuelle de cette dernière (Cantú, 2005; Manalansan IV, 2005; Siraj, 2006).

Dans une plus large mesure, nous retenons que le concept de famille est omniprésent dans les écrits sur l'ethnicité au sein des études LGBTQ+. Selon Yuval-Davis (1992) et Valenzuela (1999), la famille est un lieu primaire qui non seulement offre à la personne migrante un soutien émotionnel et/ou matériel lui permettant de faire face à la discrimination, mais également comporte certaines obligations que la personne migrante doit accomplir envers ses membres. Apprendre à s'en distancier et apprendre à développer son autonomie émotive et financière se posent, dans la majorité des cas, comme étant des stratégies nécessaires à l'affirmation d'une identité sexuelle non normative (Acosta, 2008; Siraj, 2006).

Cependant, le fait de ne pas parler de sa sexualité à ses parents ou à sa famille n'est pas nécessairement considéré comme une forme de discrimination, mais peut aussi être interprété comme une forme de négociation nécessaire au maintien des liens familiaux. 
Decena (2008) qualifie cette stratégie par la figure du "sujet tacite ». Cette figure qui vient rompre avec la dichotomie visibilité/invisibilité ou encore dicible/indicible, qui est au cour de l'image du coming-out, propose la possibilité "de demeurer dans le placard " sans y être restreint. L'homosexualité n'est pas complètement dissimulée, ni ouvertement exposée. Elle est vécue et ressentie sans devenir le marqueur principal de l'identification du sujet; elle est tacite. Le silence favorise également parfois la protection de l'honneur, de la respectabilité et du pouvoir ou encore la protection de certains individus qui souhaitent avoir des relations sexuelles avec des personnes du même sexe sans nécessairement revendiquer une identité homosexuelle (Acosta, 2008; Calixte, 2005; Chahine, 2008)

Une autre stratégie souvent évoquée dans la littérature portant sur les réalités LGBTQ+ en contexte migratoire est liée à la conformité de genre (Acosta, 2008; Chahine, 2008; Peña, 2005). En effet, ces études montrent que certaines personnes homosexuelles qui évitent des comportements transgressifs en matière de genre ont plus de facilité à conserver des liens cordiaux avec leur famille. Malheureusement, les personnes queer qui ne répondent pas aux attentes de genre auront souvent tendance à se retirer ou à se distancier de leur famille ou de leur communauté ethnique (Chahine, 2008). Cette distance de la famille ou de la communauté ethnique amène parfois certaines personnes à s'impliquer au sein de diverses communautés queer dans leur pays d'établissement. Cependant, cette implication ne se fait pas nécessairement sans difficultés (Anzaldúa, 1999; Randazzo, 2005). En effet, Espin (1997) souligne que certaines personnes queer racisées se retrouvent souvent prises entre le racisme de la société dominante et les attentes sexistes de leurs communautés dites d'attache. Certains auteurs parlent même de dichotomie identitaire ressentie par les personnes queer racisées, celles-ci devant choisir entre l'une ou l'autre facette de leur identité (Ambrosi, 2005; Fygetakis, 1997). Néanmoins, les communautés queer dans les pays d'établissement demeurent souvent les principaux lieux de soutien et permettent à plusieurs personnes de développer et de maintenir des réseaux significatifs et de consolider leurs identifications ethniques et sexuelles (Acosta, 2008; Chbat, 2011; Roy, 2013). D’ailleurs, ces marginalisations face à la communauté ethnique et à la communauté gaie et lesbienne majoritaire conduisent à la création de nouveaux espaces post-migratoires au sein desquels les personnes queer racisées peuvent élargir et redéfinir les frontières de leurs identifications multiples (Acosta, 2008; Chbat, 2011). Ces espaces deviennent souvent des lieux de partage de la langue et de la sexualité et représentent pour plusieurs de nouvelles familles (Acosta, 2008; Manalansan IV, 2005; Randazzo, 2005; Smith, 2005). 


\section{Le cas particulier de Helem}

"Helem est une

organisation non

gouvernementale

$(O N G)$ qui a pris

naissance à Beyrouth

(Liban) et qui milite

publiquement pour

les droits des per-

sonnes $L G B T Q+$. "

Helem est une organisation non gouvernementale (ONG) qui a pris naissance à Beyrouth (Liban) et qui milite publiquement pour les droits des personnes LGBTQ+. Cette organisation a été mise sur pied en 2001 par un groupe d'hommes scolarisés, urbains et professionnels appartenant à une classe socioéconomique moyenne relativement homogène (Chahine, 2008). Cependant, ce qui s'apparentait initialement à une organisation locale, comprenant une quarantaine de membres sur papier, est devenu en l'espace de quelques années un groupe important soutenu par des réseaux à l'échelle internationale. Les groupes appuyant Helem sont situés à Montréal (Canada), Paris (France), Sydney (Australie) et San Francisco (États-Unis). L'étendue de Helem, maintenant établie dans divers continents et composée de nombreux membres appartenant majoritairement à la vaste diaspora libanaise, met en lumière les dynamiques transnationales qui sont constitutives de cette organisation (Chahine, 2008). Les objectifs politiques de Helem, qui visent notamment l'annulation de la loi libanaise (article 534 du Code pénal) punissant toutes relations sexuelles jugées " non naturelles ", sont mis en œuvre par la réalisation de diverses activités liées à une stratégie de visibilité accrue (Chahine, 2008). Bien que cette stratégie suive la logique prédominante des mouvements des droits civiques occidentaux, cette visibilité devient, dans le cas de Helem, une condition nécessaire à la validation des droits des personnes LGBTQ+ et, dans une plus large mesure, un outil d'empowerment et de changement social (Chahine, 2008; Chbat, 2011).

Si certains auteurs comme Massad (2002) critiquent Helem pour ses visées prooccidentales, d'autres comme Chahine (2008) trouvent que les organisateurs de cette ONG adoptent une position claire et nuancée lorsqu'ils parlent de visibilité de leurs membres et ne tentent pas de résoudre tous les problèmes concernant les communautés LGBTQ+ en adoptant une attitude colonialiste. En effet, les membres ne sont pas tenus de divulguer publiquement leur (homo)sexualité ou de se conformer à une identité quelconque en s'impliquant au sein de Helem. Les politiques de Helem sont sensibles au fait que plusieurs personnes ayant des relations sexuelles et/ou amoureuses avec des personnes du même sexe ne sont pas nécessairement à l'aise avec l'usage et l'appropriation des termes "gai ", "lesbienne " ou "bisexuel " pour parler d'elles-mêmes. Certaines personnes ont développé des identifications qui leur sont propres et qui sont plus adaptées aux réalités sociopolitiques dans lesquelles elles vivent. Cette variabilité des 
identifications soutient le fait que le modèle occidental du "soi », qui vise la libération et la (ré)affirmation de l'identité homosexuelle à travers notamment l'appropriation et la mise en œuvre du coming-out, ne peut pas s'appliquer à toutes les personnes queer (Chahine, 2008; Roy, 2013). Les notions de visibilité et de coming-out ne peuvent être à elles seules des marqueurs de l'identité homosexuelle. Il conviendrait davantage de les considérer sous l'angle des stratégies variables selon les individus et les contextes sociaux, culturels et économiques dans lesquels elles peuvent être mobilisées (Chbat, 2011). La visibilité de l'homosexualité ne peut donc pas être strictement comprise en opposition à l'invisibilité de l'homosexualité. Elle se démarque davantage comme un concept fluide dont la signification est en constante négociation afin de s'adapter aux besoins des contextes spécifiques (Chahine, 2008; Chbat, 2011; Roy, 2013). En bref, les discours identitaires des personnes queer racisées, notamment libanaises, ne peuvent être interprétés comme des blocs monolithiques. Il faut prendre en considération des facteurs interpersonnels et structuraux pour saisir la multidimensionnalité de ces discours, d'où la pertinence des données que nous nous apprêtons à présenter. Dans le cas précis de cet article, nous tenterons de comprendre comment l'implication à différents degrés au sein d'un organisme militant pour les droits des minorités sexuelles et raciales comme Helem a un impact sur les façons dont ces personnes articulent leurs identifications ethno-sexuelles.

\section{Cadre d'analyse}

"Historiquement conçue au sein du mouvement américain du Black Feminism dans les années 1970, lequel dénonçait le réductionnisme des approches monistes qui excluait toutes formes d'inégalités multiples de son analyse, l'intersectionnalité est aujourd'hui appliquée par un nombre grandissant de chercheures et chercheurs appartenant tous à des champs scientifiques variés..."
Le cadre d'analyse que nous avons mobilisé dans notre recherche est centré essentiellement sur le féminisme intersectionnel. Historiquement conçue au sein du mouvement américain du Black Feminism dans les années 1970, lequel dénonçait le réductionnisme des approches monistes qui excluait toutes formes d'inégalités multiples de son analyse, l'intersectionnalité est aujourd'hui appliquée par un nombre grandissant de chercheures et chercheurs appartenant tous à des champs scientifiques variés (Bilge, 2015). Il est intéressant de faire un parallèle ici. En effet, à l'image des militantes africaines-américaines qui ne se retrouvaient pas au cœur des mouvements revendicateurs post-1968, lesquels étaient segmentés et exclusifs selon des réalités 
distinctes (mouvement des femmes, mouvement gai et lesbien, mouvement noir, etc.), certains militants queer racisés ont ressenti le besoin de créer des espaces dans lesquels il était possible de conjuguer leurs luttes, ces dernières étant multiples et indivisibles. Des organismes, tels que Helem, Arc-en-ciel d'Afrique ou encore AGIR (Action LGBTQ avec les immigrantEs et réfugiéEs), ont vu le jour depuis les années 2000 en contexte montréalais afin de rendre compte et de militer en faveur des identifications multiples de ces personnes qui devaient jusqu'alors choisir de s'allier politiquement à une seule facette de leur identité.

Ainsi, à la lumière de cette approche ancrée historiquement dans des revendications politiques qui cherchent à mettre en dialogue l'intersection de diverses formes de marginalisations, il nous paraît tout à fait pertinent d'analyser les discours de nos répondantes et répondants à travers cette lentille. L'intersectionnalité vise précisément à mettre en dialogue l'analyse simultanée de plusieurs axes de structuration sociale que sont le genre, la race, l'ethnicité, la classe, la religion, le handicap, l'âge, etc. et soutient que l'impact d'un axe particulier peut varier en fonction de sa combinaison avec d'autres axes potentiels de subordination ou de privilège (Bilge, 2009; Collins, 2000). En effet, l'intersectionnalité permet non seulement d'analyser les expériences de subordination de certains individus, mais également de rendre compte des ambiguïtés et des contradictions relatives à certains privilèges (Bilge, 2015). Cette lentille analytique cherche donc à rompre avec le cumul des oppressions rencontrées dans le parcours des individus minorisés, en réfutant le cloisonnement et la hiérarchisation des grands axes de structuration sociale. Elle se concentre particulièrement sur la façon dont l'interaction des axes (re)produit des inégalités sociales et des privilèges, ainsi que sur les mécanismes mis en œuvre par les actrices et acteurs pour faire face aux diverses oppressions (Bilge, 2015; Bowleg, 2008). En d'autres termes, l'intersectionnalité ne s'apparente pas à une approche victimisante, laquelle ne prendrait pas en considération les mécanismes de résilience des personnes potentiellement minorisées sur divers axes de structuration sociale. Au contraire, en plus de prendre en considération l'imbrication dynamique des multiples axes qui interagissent sur la vie des personnes, cette lentille analytique cherche à documenter les stratégies et les forces déployées par les personnes minorisées pour faire face à l'adversité et aux oppressions interpersonnelles et structurelles (Bowleg, 2008; Collins et Bilge, 2016).

En bref, pour les fins de notre recherche, nous utilisons l'approche intersectionnelle, car elle nous permet de saisir la multiplicité et l'imbrication des rapports de pouvoir qui façonnent les discours de nos répondantes et répondants. Ainsi, du fait que notre mémoire s'inscrit dans une démarche intersectionnelle, la sexualité ne devient plus le 
vecteur central des subjectivités de nos participantes et participants. Cette démarche vise plutôt à examiner les articulations variables des divers axes de structuration sociale qui construisent et influencent les discours identitaires et les expériences personnelles des participantes et participants que nous avons rencontrés.

\section{Méthodologie}

Notre recherche porte sur l'articulation identitaire de diverses personnes queer libanaises vivant à Montréal et s'appuie sur une méthodologie qualitative. Dans le cadre de cette étude, nous avons rencontré sept personnes (cinq femmes et deux hommes) qui étaient presque toutes impliquées de près ou de loin avec Helem Montréal. Des entrevues semidirigées effectuées à l'hiver et au printemps 2011, ainsi que des séances d'observation lors de réunions mensuelles tenues par le groupe Helem à l'automne 2010 et à l'hiver 2011, ont été privilégiées pour le terrain. Les entrevues ont été enregistrées, puis retranscrites et analysées à l'aide du logiciel Nvivo.

Les critères de sélection étaient les suivants :

1) avoir plus de 18 ans;

2) être d'origine libanaise (avoir comme lieu de naissance le Liban [primo-migrante ou primo-migrant] ou avoir au moins un parent né au Liban [descendante ou descendant]);

3) vivre à Montréal (il n'était pas important que la répondante ou le répondant ait obtenu la citoyenneté canadienne au moment de l'entrevue; la répondante ou le répondant devait seulement vivre à Montréal dans le but d'y rester pour une période indéterminée);

4) s'identifier comme gai, lesbienne, bisexuel ou bisexuelle, transexuel ou transexuelle, queer ou en questionnement, ou avoir des relations sexuelles et/ ou amoureuses avec une personne du même sexe (la revendication de l'identité homosexuelle n'était pas obligatoire, mais la pratique ou le souhait d'une sexualité non normative l'était).

Finalement, le choix de nous positionner en tant que femme d'origine libanaise ouvertement lesbienne a facilité le recrutement de nos participantes et participants. En effet, notre décision de nous situer face à nos répondantes et répondants et d'affirmer notre propre identification ethnique et sexuelle a fait en sorte que ces derniers nous ont accordé (et ce, de façon explicite) un plus grand degré de confiance, considérant que nous 
partagions avec eux des réalités similaires et que nous avions une facilité à comprendre certaines nuances dans leurs discours. Bien que notre position ait grandement favorisé les liens de confiance que nous avons eu la chance d'entretenir avec nos répondantes et répondants, nous reconnaissons également que notre expérience en tant que femme, lesbienne, d'origine libanaise ne légitime pas nos connaissances ou nos compétences pour mener cette recherche. Notre rapport personnel à notre origine ethnique, notre maitrise du français, notre faible connaissance de l'arabe, notre religion, notre statut d'étudiant, etc. sont quelques facettes de notre identité qui ont eu un impact sur notre relation avec nos répondantes et répondants et qui ne peuvent être occultées. En bref, cette démarche, qui s'inscrit directement dans les traditions associées à la standpoint theory (Harding, 1991; Haraway, 1988; Smith, 1974), nous a permis d'apporter un regard réflexif plus explicite, notamment en lien avec notre rôle de chercheure/étudiante dans la construction et l'analyse de notre objet de recherche. En définitive, notre position d'insider (Johnson, 1975) a eu un impact indéniable sur les conditions de réalisation de cette recherche et celles-ci doivent être exposées comme des biais. À l'instar de Harding (1991), nous reconnaissons que la réalisation de cette recherche, dans laquelle nous partagions des identifications similaires en termes de sexualité et d'ethnicité avec nos répondantes et répondants, ne prétendait pas préconiser une mise en suspens ou un détachement de nos valeurs et biais. Nous avons plutôt cherché à rendre explicite le fait que les valeurs font partie intégrante de la construction de la connaissance et que la réflexivité permet de prendre en considération l'influence de nos valeurs et de nos biais (Bourdieu, 1993; Roy, 2013).

\section{Profil et recrutement des participantes et participants}

Sept personnes âgées entre 23 et 52 ans ayant toutes des parcours migratoires, socioéconomiques et professionnels variés ont été rencontrées. Notre échantillon comprenait cinq femmes (âgées de 23, 26, 32 et 52 ans) et deux hommes (âgés de 30 ans). Deux des cinq femmes s'identifiaient comme étant musulmanes croyantes, non pratiquantes, alors que les cinq autres participants s'identifiaient comme étant chrétiens (trois maronites, deux grecs-catholiques), allant de très croyants et très pratiquants à peu croyants et pas pratiquants.

En plus d'avoir envoyé des annonces aux quatre organisations LGBTQ des universités montréalaises, nous avons présenté notre projet de recherche lors d'une réunion mensuelle de Helem Montréal. Les objectifs de notre recherche ont été diffusés par courriel à tous les membres de Helem. Bien que l'implication de Helem ait été significative pour notre 
recrutement, il a été relativement complexe de trouver des participantes et participants pour cette recherche. En effet, il a été difficile de diversifier notre bassin, car, en raison de son caractère privé et de ses implications personnelles, notre sujet de recherche a pu faire fuir certains répondants et répondantes potentiels. Somme toute, notre échantillon ne constitue pas un échantillon statistique, en ce sens qu'il ne représente pas un pourcentage particulier de la « communauté libanaise homosexuelle » à Montréal, cette communauté n'étant visiblement pas identifiable dans les faits et étant constituée d'individus qui ne partagent pas les mêmes définitions de l'homosexualité et de l'ethnicité.

\section{Analyse des résultats}

\section{Identité (homo)sexuelle : modèles variables, nature inchangeable}

\section{a) La sexualité tacite, mais pas nécessairement oppressante}

"Le récit traditionnel du coming-out, dans lequel la personne homosexuelle révèle sa sexualité non normative à ses parents et à son entourage, n'est donc pas partagé par tous les répondants et répondantes, qui ont trouvé des façons différentes de faire connaître ou pas leur sexualité à leur entourage." "
Comme nous en avons discuté dans la problématique, nos résultats sous-tendent que presque tous les répondants et répondantes vivent leur sexualité de façon tacite. Leur homosexualité est donc souvent sue (ou fortement soupçonnée) par leur entourage, mais pas nécessairement discutée ou nommée (Decena, 2008; Roy; 2013). Certains participants et participantes ont préféré parler directement de leur sexualité à certains membres de leur famille. Par contre, d'autres ont compris que des membres de leur famille sont au courant, mais préferent ne pas mettre des mots sur leur expérience. Le récit traditionnel du coming-out, dans lequel la personne homosexuelle révèle sa sexualité non normative à ses parents et à son entourage, n'est donc pas partagé par tous les répondants et répondantes, qui ont trouvé des façons différentes de faire connaître ou pas leur sexualité à leur entourage.

"C'est su oui, mais c'est su sans rien dire. C’est su comme déjà par le fait que je vivais avec ma copine au Liban. Et puis, j'ai su de par une amie à moi et à ma mère qu'ils en ont discuté et puis qu'elle sait, qu'elle savait en fait. Mais on n'a jamais eu de discussion! » (Dani, 32 ans) 
"...malgré les différentes façons de vivre sa sexualité, presque tous les répondants et répondantes perçoivent le coming-out aux autres comme un acte libérateur, un idéal à atteindre."

Néanmoins, malgré les différentes façons de vivre sa sexualité, presque tous les répondants et répondantes perçoivent le coming-out aux autres comme un acte libérateur, un idéal à atteindre. Cependant, la sortie du placard ne doit pas être accomplie à tout prix et doit se faire en respectant certaines conditions, par exemple ne pas mettre en danger la personne elle-même ou la famille de celle-ci et ne pas engendrer de situations qui pourraient être défavorables à leur endroit.

"C'est comme s'ils disent ok, on sait, mais on ne parle pas [...] Tu comprends, parce qu'à Beyrouth, c'est l'esprit collectif qui domine, on n'est pas à Montréal. Tu ne vas pas voir un voisin montréalais qui dit à son autre voisin : «Ta fille est lesbienne.» Chez nous, c'est une grosse insulte, ça fait un scandale. Alors, moi, je n'ai pas envie de blesser ma famille. C'est moi personnellement, moi, ils vont m'aimer et m'accepter [...] Je suis à l'étranger, pas de problème, et ma famille, elle est protégée de moi et elle ne risque pas le déshonneur, les propos des gens complètement arriérés. " (Sara, 32 ans)

Le sujet tacite amène donc les participantes et participants à se positionner face au coming-out. Pour la majorité d'entre eux, il ne faut pas nécessairement parler de sa sexualité à tous. Il est préférable de conserver parfois une certaine distance ou d'être discret lorsque la révélation de l'homosexualité peut entraîner des conséquences négatives importantes. Pour ces participantes et participants, la sexualité se vit donc davantage comme une quête intérieure, une facette de soi qu'ils désirent accepter personnellement et pas nécessairement publiquement. La divulgation est un plus, mais pas une nécessité absolue ou une priorité. Le coming-out est donc davantage associé à une honnêteté envers soi-même qu'à une obligation de se révéler aux autres.

"Moi, je ne suis pas nécessairement un militant du coming-out. Par contre, je pense que du moment que, nous, on l'a accepté, on n'est pas nécessairement obligé de le dire aux autres. En autant qu'on vit bien la situation. Il y a des endroits ou des situations dans lesquels il vaut mieux ne pas le dire. » (Riggo, 30 ans) 


\section{b) L'(homo)sexualité, un trait naturel et inchangeable de l'identité}

"Bien que les participantes et participants sentendent pour dire qu'il existe une multitude de façons de vivre son (homo) sexualité et que le coming-out n'est pas un passage obligé pour atteindre une forme d'émancipation, tous expliquent leur homosexualité par des arguments biologiques et naturels. "
Bien que les participantes et participants s'entendent pour dire qu'il existe une multitude de façons de vivre son (homo) sexualité et que le coming-out n' est pas un passage obligé pour atteindre une forme d'émancipation, tous expliquent leur homosexualité par des arguments biologiques et naturels. Ce consensus s'explique notamment par le fait que tous les répondants et répondantes ont eu de la difficulté à accepter leur homosexualité et qu'ils ont tous lutté à un moment ou à un autre pour essayer de changer.

"À l'adolescence, je commençais à le sentir. J'ai vraiment essayé de faire autrement. J'ai essayé de m'intéresser aux filles, mais je n'y arrivais pas [...] Je ne pouvais pas faire semblant. C'était impossible. » (Jade, 30 ans)

" J'ai commencé à comprendre petit à petit la différence, que j’étais attiré par les hommes, sauf que je l'ai renié très longtemps à cause de tout mon bagage culturel, à cause de ma religion, etc. [...] Je me mentais parce que, dans le fond, je suis comme ça et, quelque part, je le savais. " (Riggo, 30 ans).

" La raison pour laquelle je suis en paix avec moi-même aujourd'hui, c'est parce que je sais que j'ai tout essayé pour ne pas être lesbienne, et c'était très difficile. Mais je ne peux pas. Je sais que je suis comme ça, car Dieu m'a créée ainsi. "(Carole, 26 ans)

Après plusieurs tentatives pour être "normal ", adopter une explication externe à sa volonté semble permettre de faire la paix avec soi-même. Le discours naturaliste dans le processus d'acceptation personnelle est donc essentiel dans la construction de l'identification sexuelle des participantes et participants rencontrés. L'adoption d'une explication naturelle est salvatrice et permet de réconcilier la personne avec ses échecs, avec les épisodes de désolation et de haine qu'elle a pu ressentir envers elle-même en constatant que ses désirs ne répondaient pas aux attentes normatives de sexualité. D'ailleurs, à l'instar de Chamberland (2012), les discours biologiques sur l'homosexualité sont majoritaires à l'heure actuelle. Ils semblent avoir teinté massivement les représentations sociales et 
tendent à ramener la sexualité à des manifestations de la nature, c'est-à-dire déterminées, pour une large part, par des forces innées qui échappent à la volonté individuelle et aux influences sociales (Chamberland, 2012). Cette idée soutenant que l'orientation sexuelle ne soit pas un choix est donc apaisante, car elle met un frein aux pressions sociales qui peuvent être exercées à l'endroit des personnes qui ont des attirances pour les personnes $\mathrm{du}$ même sexe (Chamberland, 2012). D'ailleurs, l'aura scientifique qui renforce la crédibilité d'un tel argumentaire agit souvent comme une fondation pour construire une rhétorique, tant pour les luttes politiques que pour les discours identitaires, et incite ultimement à un appel à la tolérance pour protéger l'état d'innocence des personnes homosexuelles qui n'ont pas choisi de naître ainsi (Chamberland, 2012). Les conceptions dominantes sur l'homosexualité soutenant que celle-ci ne relève pas du choix individuel sont donc largement partagées par nos répondantes et répondants, qui avancent qu’il leur est impossible de modifier leur sexualité.

\section{Quelques stratégies pour mieux négocier et vivre son (homo)sexualité}

\section{a) Répondre aux attentes normatives de genre}

"...certains répondants et répondantes nous ont dit qu'ils ont tendance à modifier leurs comportements selon les contextes dans lesquels ils se trouvent. Sils se conforment parfois aux attentes normatives de genre, leur famille et leur entourage semblent moins gênés par leur sexualité non normative et ne cherchent pas à leur poser des questions ou à faire des commentaires négatifs à leur endroit."
La sexualité tacite à laquelle fait référence la majorité des répondantes et répondants renvoie à une question d'interprétation et implique que leur famille et leur entourage soient en mesure de reconnaître et de décoder leur homosexualité. Plusieurs répondantes et répondants partagent l'idée que l'homosexualité devient plus facilement identifiable lorsque la personne a des comportements qui ne répondent pas aux attentes normatives de genre. Ainsi, certains répondants et répondantes nous ont dit qu'ils ont tendance à modifier leurs comportements selon les contextes dans lesquels ils se trouvent. S'ils se conforment parfois aux attentes normatives de genre, leur famille et leur entourage semblent moins gênés par leur sexualité non normative et ne cherchent pas à leur poser des questions ou à faire des commentaires négatifs à leur endroit.

"Une femme qui est très masculine, c'est toujours un dilemme de dire, comment on va la présenter [aux gens] dans la société sans les choquer? Alors 
je dirais que je suis plus neutre maintenant. Les gens ne peuvent pas vraiment juger que je suis nécessairement homosexuelle par mon apparence. » (Marta, 52 ans)

Nous retenons de cette stratégie que l'homosexualité est moins problématique lorsqu'elle est invisibilisée ou atténuée. En répondant davantage aux attentes normatives de genre, certains répondants et répondantes se sentent plus libres d'être queer face à leur entourage, car leur présentation de soi plus normative ne les réduit pas à leur homosexualité, celle-ci étant souvent associée à une transgression des attentes normatives de genre. Certains acteurs et actrices sont donc conscients du caractère malléable et performatif du genre et préferent l'adapter selon les contextes dans lesquels ils se trouvent afin de conserver souvent de meilleures relations avec leur entourage.

\section{b) La distance physique et l'autonomie financière}

"L'autonomie financière et la distance physique etlou émotionnelle sont, selon les informateurs et informatrices, les principales stratégies qui leur permettent de construire et de vivre leurs identifcations homosexuelles. "
L'autonomie financière et la distance physique et/ou émotionnelle sont, selon les informateurs et informatrices, les principales stratégies qui leur permettent de construire et de vivre leurs identifications homosexuelles. En effet, tous les répondants et répondantes estiment que l'indépendance financière est nécessaire à l'affirmation de leur identification homosexuelle et que la distance physique et/ou émotionnelle, souvent facilitée par l'immigration, favorise cette affirmation. Cependant, cette distance n'est pas toujours facile à gérer. Parfois, elle implique une rupture avec des êtres chers et contribue à la perte d'un réseau social et à la diminution d'un certain capital économique qui n'auraient pas lieu, si les informateurs et informatrices ne considéraient pas l'immigration (dans certains cas) ou l'éloignement comme les seules solutions à une vie homosexuelle au moins partiellement publique.

" J'attends juste le moment d'avoir mon indépendance financière. Ça c'est crucial. Maintenant, si tu es toujours avec eux, c'est sûr qu'il va y avoir des problèmes, des questions, des pressions. "C'est quand que tu te maries?» Donc, oui, j'ai hâte d'avoir mon indépendance, mon appart, etc. " (Jade, 30 ans)

" Je respecte mes amies libanaises qui vivent là-bas et qui sont en train de tout faire pour changer la société, mais, moi, je n'ai pas envie de m'investir comme ça [...] mais elles ne savent pas à quel point ici je suis seule. [...] 
Elles ont leurs parents à côté d'eux, la maman qui cuisine. Mais en même temps, elles ne sont pas libres. Moi, je me sens libre, mais j'en paie le prix aussi. » (Sara, 32 ans)

\section{c) Les réseaux sociaux et le milieu associatif : des espaces d'échanges sécuritaires}

Bien que l'immigration et la distance physique et/ou émotionnelle aient parfois été vécues comme des ruptures avec le passé, elles conduisent souvent les participantes et participants à faire usage d'Internet pour développer un certain réseau social et/ou s'impliquer dans des organisations gaies de Montréal (notamment Helem) dans lesquelles ils sont en mesure de développer de nouvelles relations significatives. Dans un premier temps, Internet représente, sans conteste, un réseau privilégié par nos répondantes et répondants, à travers lequel ils réussissent à établir des liens importants et à avoir accès à plusieurs ressources et informations. Certains d'entre eux ont consulté à plusieurs reprises des groupes de discussion sur Internet lorsqu'ils avaient des questionnements sur leur orientation sexuelle avant de s'impliquer au sein d'une association telle que Helem. Dans un deuxième temps, les associations LGBTQ+ leur permettent de se retrouver avec des personnes qui, à leur avis, partagent des réalités ethno-sexuelles similaires. Dans certains cas, l'implication au sein de ces organisations leur a permis de sortir de l'isolement et leur a fourni un espace physique au sein duquel ils ont établi des liens significatifs tant au niveau professionnel que personnel.

«Depuis que je suis à Helem, j’ai doublé mes amis et j'ai trouvé des personnes avec qui je ressens plus d'affinités. Des amis d'origine arabe, oui, il y a un certain degré d'appartenance parce que, oui, on partage un peu le même style de vie, on a les mêmes parents. On est en train de vivre les mêmes difficultés. » (Jade, 30 ans)

"C'est sûr que je suis plus moi-même avec les amis gais parce que je n'ai rien à cacher et on peut se parler des vraies choses. Je n'ai pas peur qu'un commentaire soit pris de travers. " (Riggo, 30 ans)

Limplication au sein d'organismes communautaires agit donc positivement sur l'articulation identitaire des participantes et participants rencontrés. Pour plusieurs, cette implication leur permet de consolider des amitiés et de développer des relations qui s’apparentent à de nouvelles familles. Les répondantes et répondants avancent pour la 
plupart se sentir compris par leurs pairs. En plus d'agir comme un espace sécuritaire pour (re)négocier leur identification ethno-sexuelle, Helem représente un soutien émotionnel pour certaines personnes qui se sentent rejetées par leur entourage.

«Pour moi, Helem, c'est un organisme qui aide les gens qui ont des difficultés avec leur identité, avec leur communauté et leurs amis et leurs familles à cause de leur orientation sexuelle. Helem a été là pour moi. J'ai eu la chance d'être entourée de personnes qui ont pris soin de moi. Je n'aurais pas réussi sans eux. J'ai été au fond de la dépression et j'ai même pensé au suicide. " (Carole, 26 ans)

Cependant, tous les répondants et répondantes ne sont pas impliqués au même titre au sein de Helem, car, pour certains, il semblait important de diversifier leur réseau social et de ne pas seulement se retrouver entre "Libanais » ou entre " gais ». En outre, le militantisme pour certains membres s'avère une tâche complexe, car elle est faite en secret de leur famille et/ou de leur réseau non homosexuel. Lors des réunions mensuelles, certains ont mentionné le fait qu'ils cachaient à leurs parents leur implication ou l'existence d'une telle association. L'implication envers Helem (en tant que membre ou administrateur) semble donc être une activité sécuritaire au sein de laquelle certaines personnes développent des relations significatives et peuvent négocier leur identification ethno-sexuelle avec de nouveaux paramètres, mais demeure également, dans certains cas, une facette cachée de leur identité.

\section{Identification ethnique : rapport ambivalent au Liban et à la « communauté libanaise »}

Ces espaces de rencontres auxquelles participent certains de nos répondants et répondantes ne sont pourtant pas à l'épreuve de contradictions et de tensions. En effet, ces espaces d'échanges lient des individus venant de milieux socioéconomiques variés et ayant des convictions religieuses et sociales potentiellement conflictuelles. À cet égard, les différents discours qui se rapportent à l'identification ethnique de nos participantes et participants mettent bien en lumière le double rapport de domination qui s'établit simultanément entre les frontières qui distinguent ceux qui appartiennent au groupe ethnique et ceux qui en sont exclus (Juteau, 1999). Nous constatons que les sept participantes et participants vivent inégalement leur identification ethnique. Pour plusieurs, les rapports qu' ils entretiennent avec la " communauté " libanaise sont ambivalents, car il semble difficile, selon eux, de revendiquer leur identité libanaise face à une communauté dans laquelle ils se sentent 
rejetés et jugés. Plusieurs d'entre eux décrivent les membres de la communauté libanaise comme "fermés d'esprit » et " hyprocrites".

"Ils ne sont pas ouverts d'esprit. Ils ne sont pas ouverts au changement, à la diversité. Ils sont juste dans leur bulle et ils pensent que ce qui est bon dans leur esprit est ce qui est bon en général. » (Léa, 23 ans)

Malgré plusieurs frustrations envers la " communauté » libanaise, principalement dues au fait qu'ils ne se sentent pas acceptés en tant qu'homosexuels, tous les répondants et répondantes entretiennent également des sentiments ambivalents envers cette dite communauté au sein de laquelle ils estiment retrouver des comportements et des valeurs uniques qui les rendent fiers d'être Libanais.

"Je suis fière de plusieurs choses que nous partageons dans notre communauté. Le respect, la chaleur, la gentillesse envers les autres. Nous avons une façon de penser comme, par exemple, si quelque chose arrive à quelqu'un, nous penserons à le contacter et prendre soin de lui. J'ai des amis qui appartiennent à d'autres ethnies, et ce n'est pas la même chose. Ils sont plus froids, mais les amis que j'ai, qui sont Libanais, sont plus chaleureux. Même si nous sommes hypocrites, nous sommes quand même gentils et nous nous soucions des autres et nous aimons notre culture. » (Carole, 26 ans)

Tantôt hypocrite, tantôt chaleureuse et unique, la " communauté " libanaise dont parlent nos répondantes et répondants s'apparente à une communauté fixe, relativement homogène, composée de membres qui s'attribuent des traits distinctifs fondamentaux correspondant à des valeurs essentielles qui leur viennent de leurs traditions culturelles (Barth, 1969). Ces traits distinctifs fondamentaux correspondent à des schèmes ou à des catégories générales au nom desquels les membres du groupe peuvent se reconnaître ou pas : " être de grands voyageurs, être généreux et chaleureux, être fiers et organisés ». Il se dégage implicitement de cette représentation de la " communauté " libanaise la notion de frontière qui délimite le groupe de façon dynamique (Barth, 1969; Juteau, 1999). Parfois, certains de nos répondants et répondantes se sentent inclus (et s'incluent) au sein de cette dite "communauté ", parfois, ils s'en détachent ou se sentent rejetés de cette dernière, car ils ne répondent pas aux attentes normatives de genre ou de sexualité qui sont partagées par la majorité des membres de ce groupe.

"Je n'aime pas que tout soit attribué aux Arabes. Je ne pense pas que nous, les Libanais chrétiens, nous sommes Arabes. Nous sommes, c'est une grande 
histoire le Liban. Les Phéniciens, c'est autre chose que les pays arabes, c'est différent. Moi je pense que ces pays-là ne veulent pas se développer. Le Liban est différent. Donc, moi, je ne veux pas être associée à être Arabe. Je n’ai pas de problème avec le Moyen-Orient. Peut-être disons que j’aimerais plus dire que je viens de la Méditerranée. " (Dani, 32 ans)

La citation précédente souligne le fait que certains répondants et répondantes ne partagent pas les valeurs ou les comportements qu'ils associent à la "communauté " libanaise et préferent plutôt s'en dissocier et s'identifier à la " communauté blanche ". Cette stratégie de distanciation vis-à-vis d'une catégorie stigmatisée (être Arabe équivaut au fait d'être musulman) réitère le fait que l'ethnicité a la propension d'être à la fois une catégorie manipulée et une catégorie de manipulation (Kilani, 2000). Cependant, nous sommes également consciente que, dans le cas particulier de cette participante, certains facteurs légitiment plus facilement sa revendication à la culture dominante (ou non orientale), par exemple sa religion (chrétienne), son lieu de naissance (Montréal), son apparence physique (peau pâle) et sa maîtrise du français. Ceci étant dit, plusieurs répondantes et répondants partagent des représentations de la " communauté » libanaise qui sont construites, d'une part, par référence à des représentations hégémoniques et unilatérales de l'Autre non occidental et, d'autre part, par référence à des frontières claires impliquant une nette démarcation entre le "nous " et le " non-nous " (Bilge, 2010). Une des représentations les plus marquantes de l'Autre non occidental est l'altérité musulmane (Bilge, 2010). Cette dernière devient une forme d'unité contingente à l'identité et aux valeurs européennes/occidentales (Bilge, 2010; Yegenoglu, 2006). Cette stratégie cherchant à dissocier les Arabes chrétiens des Arabes musulmans permet de lier ces premiers avec l'Occident moderne (Naber, 2010). Les catégories "Arabes" et "Canadiens/Occidentaux » sont donc mutuellement constitutives et existent toutes deux en union dans le contexte d'immigration et de racialisation canadien (Naber, 2010). Ainsi, les représentations binaires partagées par certains de nos répondants et répondantes présentent l'Occident comme un espace résolument féministe, sympathique à la cause des homosexuelles, tolérant et libéré sur le plan sexuel, alors que l'Orient (le Liban musulman) est intrinsèquement sexiste et homophobe (Bilge, 2010; Roy, 2013). Sans chercher à dévaloriser les expériences oppressantes qu'ont vécues plusieurs de nos participantes et participants au sein de leur famille et de leur communauté, nous constatons que la majorité d'entre eux ont intériorisé un certain discours orientaliste qui s'appuie sur des oppositions hiérarchiques entre l'Occident et l'Orient et qui naturalise l'infériorité de 
l'Orient (surtout des musulmans) en y voyant une entité monolithique, rétrograde et étrange (Bilge, 2010; Saïd, 1978).

Soulignons cependant que ce discours n'est pas partagé par tous les participants et participantes et que certains d'entre eux parlent du Liban de façon positive, sans faire référence aux différences religieuses.

"Chez les Orientaux, il y a une notion collective surtout de la famille qu'on n'a plus ici, qui est mille fois plus civilisée, plus moderne et humaine, selon moi. Je dis toujours à mes parents que je vis dans une société moderne, mais pas nécessairement plus civilisée qu'au Liban. Après on a des valeurs différentes, on voit les choses différemment, mais je ne peux pas dire que le Canada est un pays plus civilisé que le Liban. » (Sara, 32 ans)

"...malgré le fait que les participantes et participants partagent des discours différents et parfois ambivalents envers la "communauté" libanaise, tous s'entendent pour dire que les associations LGBTQ+ ethniques, notamment Helem, sont bénéfiques, car elles permettent de négocier leur identification ethnique avec plus d'agentivité." "
En bref, malgré le fait que les participantes et participants partagent des discours différents et parfois ambivalents envers la "communauté " libanaise, tous s'entendent pour dire que les associations LGBTQ+ ethniques, notamment Helem, sont bénéfiques, car elles permettent de négocier leur identification ethnique avec plus d'agentivité. Malgré les tensions potentielles liées aux différentes religions, aux classes, au sexe, à l'âge et au statut d'immigration (pour ne nommer que ces facteurs) au sein de la " communauté " libanaise plus vaste, tous les participants et participantes reconnaissent l'impact positif et unificateur des associations LGBTQ+ ethniques telles que Helem. Pour certains, cette association en particulier représente maintenant une nouvelle famille et leur a permis de renégocier leur rapport à leur ethnicité avec plus de liberté et de concilier leur identification (homo)sexuelle et leur identification libanaise longtemps intériorisées comme étant incompatibles et antagonistes.

«Il y a quelques années, je suis allée à la parade gaie et j'ai vu le drapeau du Liban. J'étais vraiment surprise. J'ai vu sur le char quelque chose qui était écrit et qui m’a marquée pour toujours. C'était écrit : «Manek wahdek, manik wahdik» (Tu n'es pas seul, tu n'es pas seule). Et ça m’a juste vraiment touchée parce que je sentais que je n'étais pas anormale et pas seule. J'étais capable de voir toutes ces personnes canadiennes qui étaient homo, mais ça, c'est parce 
qu'elles ont été élevées comme ça. Mais, pour moi, de voir des personnes de ma culture et de voir qu'elles étaient fières de qui elles sont, ç’a tout changé pour moi. " (Carole, 26 ans)

\section{Conclusion}

"...que les diverses conceptions de "bien vivre son homosexualité" vont au-delà du simple choix individuel entre parler de sa sexualité ou la taire."

En conclusion, nous retenons de nos entretiens que les diverses conceptions de "bien vivre son homosexualité " vont au-delà du simple choix individuel entre parler de sa sexualité ou la taire. Certains des témoignages soulèvent des confrontations potentielles ou réelles qui éclairent des relations asymétriques de pouvoir. En effet, les témoignages que nous avons recueillis mettent de l'avant des personnes qui refusent d'afficher leur homosexualité en toutes circonstances, par volonté de ne pas être réduites à leur homosexualité. Ces personnes sont en mesure de réfléchir sur les façons qu'elles choisiront de privilégier pour parler ou non de leur sexualité non normative et ne se perçoivent pas nécessairement comme des êtres oppressés. Les répondantes et répondants ne dénigrent pas systématiquement l'affichage public de leur sexualité dans le contexte actuel, mais ils jugent qu'il est plus important pour eux, en tant qu'individus d'origine libanaise, de considérer pleinement l'importance de leur réseau social avant de sortir du placard. De plus, il est possible d'être " out » dans un domaine d'activités sociales ou dans un réseau social particulier, tout en étant dans le placard dans d'autres champs et auprès d'autres groupes. Le coming-out n'est donc pas synonyme de libération pour tous et même, inversement, le fait de ne pas divulguer son identification homosexuelle peut parfois se traduire par plus d'aise et de privilèges. Cependant, bien que les politiques de visibilité et de divulgation varient selon les contextes dans lesquels se trouvent les participantes et participants, la majorité d'entre eux reconnaissent les impacts positifs que leur confere une implication, même partielle, au sein d'un organisme communautaire comme Helem. Bien qu'il ne soit pas à l'abri de tensions politiques et religieuses, ce dernier représente un lieu physique et symbolique au sein duquel les participantes et participants peuvent articuler leurs identifications multiples, voire redéfinir, dans certains cas, de nouvelles façons de construire et de se (re) présenter ce que leur (homo)sexualité et leur ethnicité évoquent pour eux personnellement.

En outre, d'un point de vue analytique, nous constatons également que la lentille intersectionnelle nous a permis de dresser un portrait nuancé et non monolithique 
des diverses subjectivités à l'étude. Cependant, son application concrète demeure complexe, notamment dans la mesure où il est parfois ardu de prendre en considération l'influence simultanée de nombreux axes de structuration sociale sur les discours des personnes rencontrées. De plus, il s'avère primordial de ne pas tomber dans le piège d'une surinterprétation fondée sur nos préceptes théoriques plutôt que sur nos données empiriques (Bilge, 2009).

Finalement, nous souhaitons souligner quelques directions possibles pour de futures recherches qui permettront de vérifier et compléter nos résultats. Une première piste prometteuse serait d'agrandir l'échantillon d'enquête, en incluant notamment plus d'hommes, étant donné que notre échantillon n'en comprenait que deux. De plus, il serait intéressant de recruter des participantes et participants qui ne sont pas affiliés à l'association Helem pour comparer les expériences des individus qui sont impliqués dans d'autres associations communautaires. Aussi, une étude longitudinale pourrait apporter des dimensions enrichissantes, nous permettant de mieux saisir les divers contextes et les différents parcours migratoires dans lesquels évoluent les personnes LGBTQ+ d'origine libanaise à Montréal. Finalement, l'exploration en profondeur d'autres axes de structuration sociale, notamment l'affiliation politique, l'âge, le degré de scolarité, le handicap, etc., apporterait plus de nuances et de raffinement à l'analyse intersectionnelle. En terminant, face à la récente montée des forces politiques conservatrices dans les pays occidentaux et non occidentaux, les discours et les stratégies de négociation des identifications LGBTQ+, notamment libanais, pourraient subir d'importants changements, d'où la nécessité de mettre à jour les conclusions tirées de projets de recherche antérieurs et d'en développer des nouveaux qui viseraient également à expliciter les subjectivités LGBTQ+ et à favoriser le bien-être de ceux qui les revendiquent.

\section{Notes}

1 La conceptualisation " occidentale » de l'homosexualité fait référence à une sexualité hautement normalisée et quasi hégémonique d'une identité gaie et lesbienne qui prône la divulgation, la visibilité et l'affichage public de l'identité (homo)sexuelle. Elle est fondée sur un mode de vie et une culture foncièrement dépolitisés, consuméristes et majoritairement composés d'individus occidentaux, blancs, jeunes, de classe moyenne (ou aisée) et urbains (Martin, 2013).

2 Le terme « queer " est mobilisé dans cet article non pas au sens anti-identitaire de sa définition tel qu'utilisé principalement au sein de la théorie queer, mais afin de rendre compte de la diversité des pratiques et des identités sexuelles qui ne peuvent être pensées à l'intérieur de catégories institutionnelles. Ainsi, le terme " queer » est mobilisé pour désigner toutes formes de sexualité qui s'inscrivent à l'extérieur du cadre hétéronormatif. 
3 Le terme « racisé » est utilisé dans cet article pour rendre compte du processus engendré lorsqu'un nombre de critères sont isolés afin de décrire les particularités humaines et les classer (Guillaumin, 1972). Un individu sera racisé lorsque des différences bio-physiques seront altérisées comme étant particulières et souvent inférieures par la société dominante (Guillaumin, 1972).

\section{Bibliographie}

ACOSTA, Katie (2008). "Lesbianas in the Borderlands: Shifting Identities and Imagined Communities ", Gender and Society, Vol. 22, № 5, p. 639-659.

AMBROSI, Sophie (2005). Identité ethnique et identité érotique : le cas des lesbiennes d'origine haïtienne, [mémoire de maîtrise], Montréal, Université de Montréal, 152 p.

ANZALDÚA, Gloria (1999). Borderlands, La Frontera: The New Mestiza, San Francisco, Aunt Lute Books, 217 p.

APPADURAI, Arjun (1996). Modernity at Large: Cultural Dimensions of Globalization, Minneapolis, University of Minnesota Press, $248 \mathrm{p}$.

BARTH, Fredrik (1969). "Les groupes ethniques et leurs frontières », dans Ph. Poutignat et J. StreiffFénart (dirs.), Théories de l'ethnicité, PUF, p. 203-249.

BILGE, Sirma (2015). "Le blanchiment de l'intersectionnalité », Recherches féministes, Vol. 28, N 2, p. 9-32.

BILGE, Sirma (2010). " ...alors que nous, Québécois, nos femmes sont égales à nous et nous les aimons ainsi : la patrouille des frontières au nom de l'égalité de genre dans une "nation» en quête de souveraineté ", Sociologie et Sociétés, Vol. XLII, printemps 2010, p. 197-226.

BILGE, Sirma (2009). "Smuggling Intersectionality into the Study of Masculinity: Some Methodological Challenges ", Feminist Research Methods: An International Conference, University of Stockholm, 4-9 février 2009.

BOURDIEU, Pierre (1993). "Comprendre ", dans Pierre Bourdieu (dir.), La misère du monde, Paris, Seuil, p. 903-925.

BOWLEG, Lisa (2008). "When black + lesbian + woman $\neq$ black lesbian woman: The methodological challenges of qualitative and quantitative intersectionality research », Sex Roles, Vol. 59, p. 312-325.

CALIXTE, Shana (2005). «Things Which Aren't To Be Given Names: Afro-Caribbean and Diasporic Negotiations of Same Gender Desire and Sexual Relations ", Canadian Woman Studies/Les cahiers de la femme, Vol. 24, No 2, 3.

CANTÚ, Lionel (2005). "Well-Founded Fear: Political Asylum and the Boundaries of Sexual Identity in the U.S.-Mexico Borderlands ", dans Eithne Luibhéid et Lionel Cantú (dirs.), Queer Migrations: Sexuality, U.S. Citizenship, and Border Crossings, Minneapolis, University of Minnesota Press, p. 61-74. 
CHAHINE, Pascal (2008). (Re)constructing Beirut: Helem and "Local" Homosexualities, [mémoire de maîtrise], Montréal, McGill University, 104 p.

CHAMBERLAND, Line (2012). "La biologie de l'homosexualité : un révélateur de l'imbrication du sexisme et de l'hétérosexisme ", dans Louise Cossette (dir.), Cerveau, hormones et sexe : des différences en question, Montréal, Les éditions du remue-ménage, p. 49-83.

CHBAT, Marianne (2011). Articulations et négociations des identifications ethno-sexuelles des gais et des lesbiennes d'origine libanaise à Montréal, [mémoire de maîtrise], Montréal, Université de Montréal, $105 \mathrm{p}$.

COLLINS, Patricia Hill, et Sirma BILGE (2016). Intersectionality, Cambridge, Polity Press, 249 p.

COLlinS, Patricia Hill (2000 [1990]). Black Feminist Thought: Knowledge, Consciousness, and the Politics of Empowerment, New York, Routledge, 336 p.

DECENA, Carlos Ulises (2008). "Tacit Subjects », GLQ, Vol. 14, № 2, p. 339-359.

ESPIN, Olivia (1997). Latina Realities: Essays on Healing, Migration, and Sexuality, Westview Press, $195 \mathrm{p}$.

FOUCAULT, Michel (1976). Histoire de la sexualité, Tome 1 : La volonté de savoir, Gallimard, 211 p.

FYGETAKIS, Leah M. (1997). «Greek American Lesbians, Identity Odysseys of Honorable Good Girls ", dans Beverly Greene (dir.), Ethnic and Cultural Diversity Among Lesbians and Gay Men, London, Sage Publications, p. 152-190.

GOSINE, Andil (2008). "FOBs, Banana Boy, and The Gay Pretenders », dans Susan Driver (dir.), Queer Youth Cultures, Albany, State University of New York Press, p. 223-242.

GUILLAUMIN, Colette (1972). L'idéologie raciste : Genèse et langage actuel, Publications de l'Institut d'Études et Recherches Interethniques et Interculturelles, La Haye, Mouton, 243 p.

HARAWAY, Donna (1988). "Situated Knowledges: The Science Question in Feminism and the Privilege of Partial Perspective ", Feminist Studies, Vol. 14, № 3, p. 575-599.

HARDING, Sandra (1991). Whose Science? Whose Knowledge? Thinking from Women's Lives, Ithaca, Cornell University Press, 319 p.

JIMENEZ, Marina (2004). "Gay Jordanian Now Gloriously Free in Canada », The Globe and Mail, May 20, 2004, A3.

JOHNSON, John (1975). Doing Field Research, New York, Free Press, 225 p.

JUTEAU, Danielle (1999). L'ethnicité et ses frontières, Presses de l'Université de Montréal, 226 p.

KHALAF, Samir, et John GAGNON (2006). Sexuality in the Arab World, Liban, Saqi Books, 312 p.

KILANI, Mondher (2000). "L'inhumanité de l'autre? Notes introductives sur quelques concepts clés », L’imbroglio ethnique en quatorze mots-clés, Éditions Payot Lausanne, Anthropologie, France, p. 931. 
LUIBHÉID, Eithne (2005). «Introduction: Queer Migration and Citizenship », dans Eithne Luibhéid et Lionel Cantú (dirs.), Queer Migrations: Sexuality, U.S. Citizenship, and Border Crossings, Minneapolis, University of Minnesota Press, p. ix.

MANALANSAN IV, Martin (2005). « Migrancy, Modernity, Mobility: Quotidian Struggles and Queer Diasporic Intimacy ", dans Eithne Luibhéid et Lionel Cantú (dirs.), Queer Migrations: Sexuality, U.S. Citizenship, and Border Crossings, Minneapolis, University of Minnesota Press, p. 146-160.

MARTIN, Marie-Ève (2013). Représentations filmiques de lesbiennes/queers issues de la diaspora indienne en Occident, [mémoire de maîtrise], Montréal, Université de Montréal, 135 p.

MASSAD, Joseph (2002). «Re-orienting Desire: The Gay International and the Arab World », Public Culture, Vol. 14, No 2, p. 361-385.

NABER, Nadine (2010). "Doing it: Sexualities, Arab American Feminities: Beyond Arab Virgin American(ized) Whore ", dans Rebecca F. Plante et Lis M. Maurer (dirs.), Doing Gender Diversity: Reading in Theory and Real-World Experiences, Westview Press, 576 p.

PEÑA, Susana (2005). «Visibility and Silence: Mariel and Cuban American Gay Male Experience and Representation », Eithne Luibhéid et Lionel Cantú (dirs.), Queer Migrations: Sexuality, U.S. Citizenship, and Border Crossings, Minneapolis, University of Minnesota Press, p. 125-145.

RANDAZZO, Thomas J. (2005). «Social and Legal Barriers: Sexual Orientation and Asylum in the United States ", dans Eithne Luibhéid et Lionel Cantú (dirs.), Queer Migrations: Sexuality, U.S. Citizenship, and Border Crossings, Minneapolis, University of Minnesota Press, p. 30-60.

ROY, Olivier (2013). « Homme immigrant cherche homme : (re)formations de subjectivités ethnosexuelles en contexte post-migratoire au Québec ", [thèse de doctorat], Montréal, Université de Montréal, $444 \mathrm{p}$.

SAÏD, Edward (1978). Orientalism, New York, Vintage Books, 345 p.

SIRAJ, Asifa (2006). «On Being Homosexual and Muslim: Conflicts and Challenges », dans Lahoucine Ouzgane (dir.), Islamic Masculinities, Londres, Zed Books, p. 202-216.

SMITH, Dorothy (1974). "Women's Perspective as a Radical Critique of Sociology ", Sociological Inquiry, Vol. 44, № 1, p. 7-13.

SMITH, Miriam (2005). «Identité queer : diaspora et organisation ethnoculturelle et transnationale des lesbiennes et des gais à Toronto", Lien social et Politiques, № 53, p. 81-92.

VALENZUELA, Abel (1999). "Gender Roles and Settlement Activities among Children and their Immigrant Families ", American Behavioral Scientist, Vol. 42, № 4, p. 720-742.

YEGENOGLU, Meyda (2006). "The Return of the Religious. Revisiting Europe and its Islamic Others ", Culture and Religion, Vol. 7, № 3, p. 245-261.

YUVAL-DAVIS, Nina (1992). «Fondamentalism, Multiculturalism and Women in Britain », dans J. Donald et A. Rattansi (dirs.), Race, Culture and Difference, Londres, Sage Publications, p. 278-291. 